\title{
Oral administration of salmon cartilage proteoglycan extends the survival of allografts in mice
}

\author{
KRISANA ASANO $^{1,2}$, EMIKO TAKAHASHI ${ }^{1}$, SAYURI YOSHIMURA ${ }^{2,3}$ and AKIO NAKANE ${ }^{1,2}$ \\ ${ }^{1}$ Department of Microbiology and Immunology; ${ }^{2}$ Biopolymer and Health Science, Hirosaki University \\ Graduate School of Medicine, Hirosaki, Aomori 036-8562, ${ }^{3}$ Tohoku Women's College, \\ Hirosaki, Aomori 036-8503, Japan
}

Received July 14, 2017; Accepted October 6, 2017

DOI: $10.3892 /$ br.2017.1011

\begin{abstract}
Proteoglycan (PG) is a complex glycohydrate that is widely distributed in the extracellular matrix. Oral administration of PG extracted from salmon nasal cartilage has been reported to attenuate the severity and proinflammatory cytokine responses in mouse experimental colitis, autoimmune encephalomyelitis, collagen-induced arthritis and obesity-induced inflammation. In the present study, the effects of salmon nasal cartilage PG on skin allografts were investigated in a mouse model. Oral administration of PG prolonged the survival of skin grafts within 10 days of transplantation. Although PG failed to inhibit allograft rejection at the final stage of transplantation, PG attenuated the cell infiltration in the skin under the transplanted site.
\end{abstract}

\section{Introduction}

Rejection is a major threat to tissue and organ transplantation, and immunosuppression is required to preserve graft function and survival (1). Although $>90 \%$ of graft survival in the majority of organ transplants survive by immunosuppression, the immunosuppressive drugs have adverse side effects on various cells and tissues. Long-term administration of the immunosuppressive compounds cause nephrotoxicity, susceptibility to infection and onset of diabetes $(2,3)$. Therefore, additional treatment options are required.

$\mathrm{CD}^{+} \mathrm{T}$ cells have long been known to be central in mediating transplant rejection (4). Acute allograft rejection

Correspondence to: Dr Krisana Asano, Department of Microbiology and Immunology, Hirosaki University Graduate School of Medicine, 5 Zaifu-cho, Hirosaki, Aomori 036-8562, Japan E-mail: krisana@hirosaki-u.ac.jp

Abbreviations: PG, proteoglycan; Tregs, regulatory T cells; EAE, autoimmune encephalomyelitis; PBS, phosphate-buffered saline; IL-17A, interleukin-17A

Key words: proteoglycan, salmon nasal cartilage, transplantation, skin graft, anti-inflammation is a T cell-dependent phenomenon and may be triggered by different types of helper $\mathrm{T}(\mathrm{Th})$ cell. Th1 cell responses initiate allograft rejection by promoting proliferation of alloreactive $\mathrm{CD}^{+} \mathrm{T}$ cells or by inducing a delayed type hypersensitivity reaction mediated by macrophages. In previous years, it has been reported that Th1 cells and Th17 cells mediate acute allograft rejection by recruiting neutrophils and monocytes into the graft, which subsequently contributes to transplant inflammation (5-7). Furthermore, it has been demonstrated that regulatory $\mathrm{T}$ cells (Tregs) induce and maintain tolerance to the allograft in experimental and clinical transplantation (8).

Proteoglycan (PG) consists of a core protein and one or more covalently attached glycosaminoglycan chain(s). It is a compound of extracellular matrix materials that exist in connective tissue, such as skin, bone, cartilage and vascular walls by forming a complex with collagen, fibronectin, laminin, hyaluronic acid and other glycoproteins. In corporation with collagen, fibronectin and laminin, PG has been demonstrated to be involved in cellular proliferation and adhesion (9). It has previously been demonstrated that PG extracted from salmon nasal cartilage exerts a potent effect on suppression of inflammatory responses induced by heat-killed Escherichia coli in mouse macrophages (10). In addition, daily oral administration of PG attenuates the severity of experimental inflammatory colitis (11), autoimmune encephalomyelitis (EAE) (12) and collagen-induced arthritis (13). Attenuation of the systemic inflammation in colitis and EAE models by daily oral administration of PG depends on suppression of Th17 lineage differentiation and an induction of Foxp $3^{+}$Treg cells $(11,12)$. Additionally, daily oral administration of PG reduced the accumulation of M1 macrophages, which induce inflammation via the production of proinflammatory cytokines, in the adipose tissue of high-fat diet-induced obesity mice (14).

In the present study, the effect of salmon nasal cartilage PG on skin graft transplantation was examined to determine whether oral administration of PG could prolong graft survival.

\section{Materials and methods}

Mice. C57BL/6 mice and BALB/c mice (age, 6-8 weeks), were purchased from CLEA Japan, Inc. (Tokyo, Japan). The mice were provided with food and water ad libitum. The C57BL/6 mice and BALB/c mice were kept separately in a 
temperature-controlled room $\left(22^{\circ} \mathrm{C}\right)$ under a $12 \mathrm{~h} \mathrm{light/dark}$ cycle and specific pathogen-free conditions at the Institute for Animal Experimentation, Hirosaki University Graduate School of Medicine (Hirosaki, Japan). All animal experiments in the present study were conducted in accordance with the Animal Research Ethics Committee of the Hirosaki University Graduate School of Medicine, and followed the Hirosaki University Guidelines for Animal Experimentation.

Preparation and administration of $P G$. Salmon nasal cartilage PG was purchased from Kakuhiro Co., Ltd. (Hirosaki, Japan). Lyophilized PG powder was dissolved in phosphate-buffered saline (PBS) to a concentration of $10 \mathrm{mg} / \mathrm{ml}$. C57BL/6 mice were administrated with $2 \mathrm{mg}$ PG per os daily. PBS served as a control.

Skin graft model. C57BL/6 recipient mice were orally administrated with PG for 10 days, then skin grafting was performed. For the experimental models of skin grafts, the procedure described by Billingham and Medawar (15) was adopted. Briefly, BALB/c donor mice were sacrificed by cervical dislocation, and $0.5 \times 0.5 \mathrm{~cm}$ sections of tail skin were removed and immersed in RPMI-1640 medium (Nissui Pharmaceutical Co., Ltd., Tokyo, Japan) supplemented with $10 \%$ fetal calf serum (JRH Biosciences, Lenexa, KS, USA). The C57BL/6 recipient mice were anesthetized with nembutal (Dainippon Sumitomo Pharma Co., Ltd., Osaka, Japan), and the fur was shaved off the dorsal trunk. At the shaved area, $0.5 \times 0.5 \mathrm{~cm}$ of skin in each recipient mouse was removed. One piece of donor tail skin was sutured to the exposed tissue of each recipient. Animals were maintained in individual cages and observed daily. PG was continuously administered daily until graft rejection was observed. In each experiment, 3-4 mice were used and three independent experiments were performed.

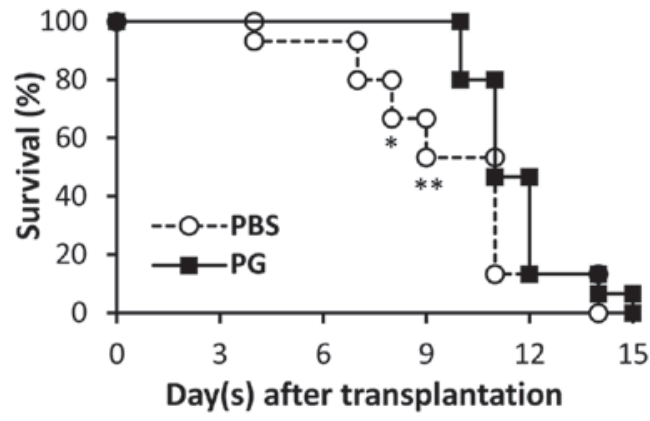

Figure 1. PG extended the survival of skin grafts. Daily oral administration of PG was initiated before skin transplantation for 10 days and continued until the graft was rejected. PBS was administered as a control. The criterion of graft rejection was a graft size $<20 \%$ of the original size. ${ }^{*} \mathrm{P}<0.05$ and ${ }^{* *} \mathrm{P}<0.01$ vs. control ( $\mathrm{n}=15$ from three independent experiments). $\mathrm{PG}$, proteoglycan; PBS, phosphate-buffered saline.

Skin graft survival. To detect graft rejection, the sizes of the grafts were recorded. The initial graft size is referred to as $100 \%$. The criterion for graft rejection was based on the graft size being $<20 \%$.

Histology. The skin tissue was collected from the graft site of C57BL/6 recipient mice. After the tissue was fixed with $10 \%$ neutral-buffered formalin (Sigma-Aldrich; Merck KGaA, Darmstadt, Germany), it was embedded in paraffin and sliced into $4 \mu \mathrm{m}$ thick sections. The sections were stained at room temperature with hematoxylin for $10 \mathrm{~min}$ and eosin for $15 \mathrm{~min}$, and observed under a BZ-X700 microscope (Keyence Corporation, Osaka, Japan). Three histologists evaluated the sections of the skin tissue.

Statistical analysis. Data are expressed as means \pm standard deviation (mean $\pm \mathrm{SD}$ ) . For graft survival, statistical analysis
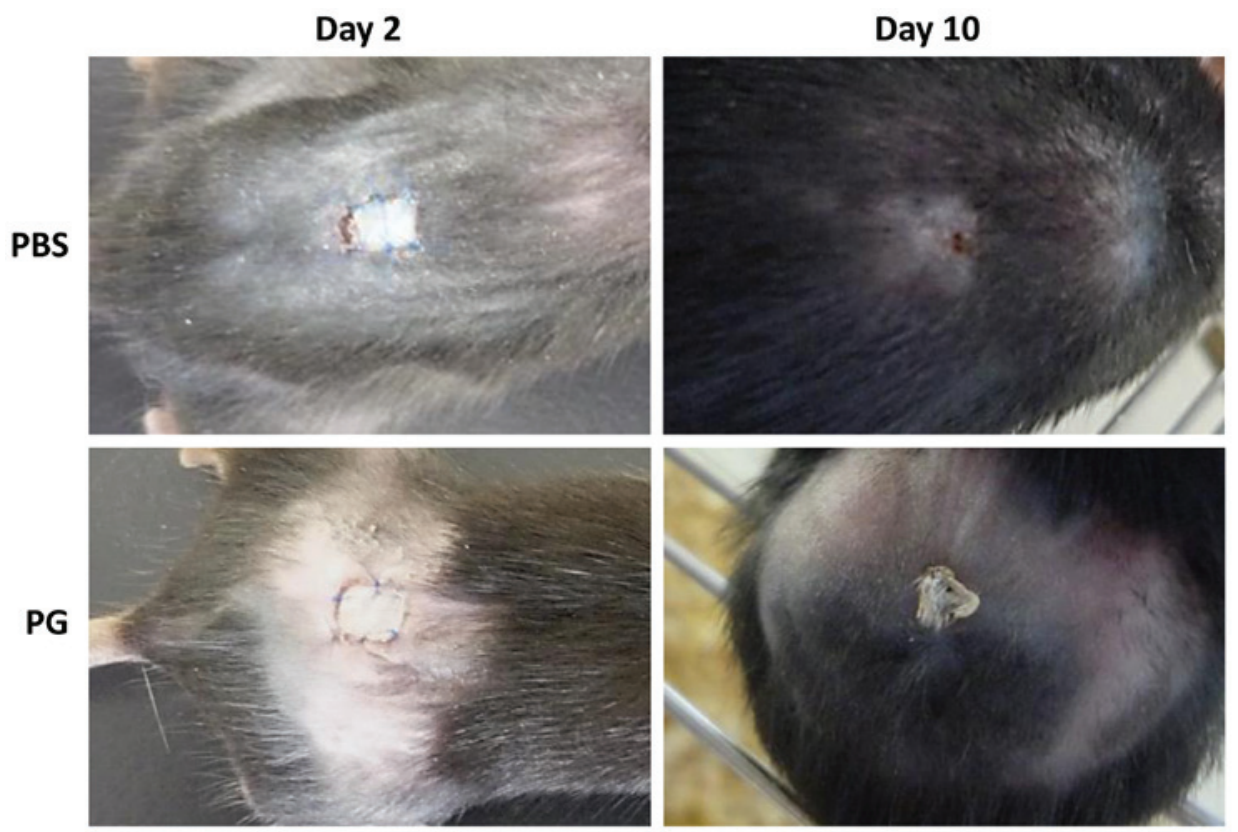

Figure 2. Macroscopic appearance of grafts in the skin-transplanted mice. The images are representatives of each group. On day 2, the grafts of the control (PBS) and PG-administered mice were retained. On day 10, the graft in the PBS-administered mouse was rejected, whereas the graft in the PG-administered mouse was retained. PG, proteoglycan; PBS, phosphate-buffered saline. 


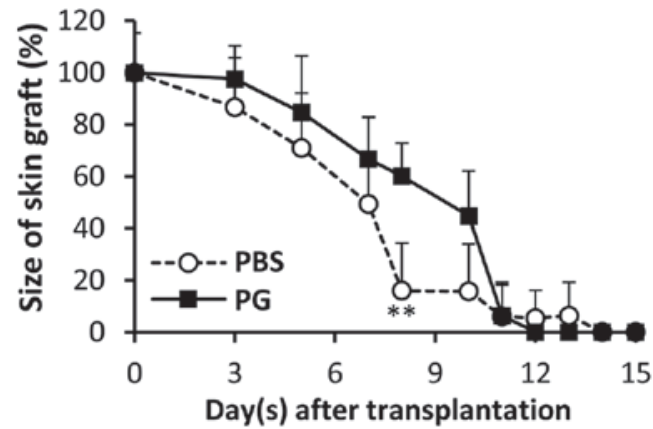

Figure 3. PG attenuated the reduction of graft size. Daily oral administration of PG was initiated before skin transplantation for 10 days and continued until the graft was rejected. PBS was administered as a control. The size of the graft was recorded ( $\mathrm{n}=12$ from three independent experiments). The initial graft size is referred to as $100 \%$. Data were expressed as means \pm standard deviation. ${ }^{* *} \mathrm{P}<0.01$ vs. control. PG, proteoglycan; PBS, phosphate-buffered saline.

was performed using Kaplan-Meier method and the statistical significance was evaluated using the log rank test. For graft size, statistical analysis was calculated using the unpaired Student's t-test. $\mathrm{P}<0.05$ was considered to indicate a statistically significant difference.

\section{Results}

Skin graft survival. Following transplantation, survival of skin grafts was observed daily. The results are presented in Fig. 1. Graft rejection was observed from day 4 in the control mice, whereas the grafts were rejected from day 10 in the PG-administered mice. Significant differences in graft survival between the control and PG-administered mice were observed on day 8 and 9. On day 15, the grafts from the PG-administered mice were totally rejected, which was identified in the control mice.

Macroscopic appearance skin grafts. The macroscopic appearance of skin grafts was observed (Fig. 2). On day 2 of transplantation, the grafts were retained in the two groups and the macroscopic appearance between the control and PG-administered mice was not significantly different. From day 3 of transplantation, the sizes of the grafts were gradually reduced and the remaining graft sizes in the control mice were smaller than those in the PG-administered mice. A significant difference between the graft sizes was observed on day 8 (Fig. 3). On day 10, the grafts of the control mice were rejected, whereas the grafts of PG-administered mice were retained (Fig. 2). Although the edge of grafts in the PG-administered mice dried, the middle region of grafts tightly adhered to the recipient skin.

Histology at graft sites. The histology of skin grafts was observed (Fig. 4). The grafts in the control mice were smaller than those in the PG-administered mice. They were loosely attached to the recipient skin. In comparison to the normal skin, the subcutaneous layer of skin graft was thickened. A reduction of cell infiltration under the graft was observed in the PG-administered mice.

\section{Discussion}

In the present study, the prophylactic effect of salmon nasal cartilage PG on skin allograft rejection was investigated. Oral administration of PG was demonstrated to attenuate the progress of skin graft rejection, although allograft rejection was not finally prevented.

Graft rejection was observed from day 4 in the PBS-administered mice, whereas the rejection in PG-administered mice was not observed until day 10 . Significant differences in graft survival between the control and PG-administered mice were identified on days 8 and 9 (Fig. 1; $\mathrm{P}=0.0177$ and 0.0034 , respectively). Reduction of graft size was correlated with graft survival (Fig. 3). Th17 cells reportedly mediate acute allograft rejection by recruiting neutrophils and monocytes into the graft, which then contribute to transplant inflammation (5-7). It is well known that interleukin (IL)-17A promotes the expression of neutrophil- and monocyte-recruiting chemokines, and the produced chemokines elicit recruitment of phagocytes $(16,17)$.

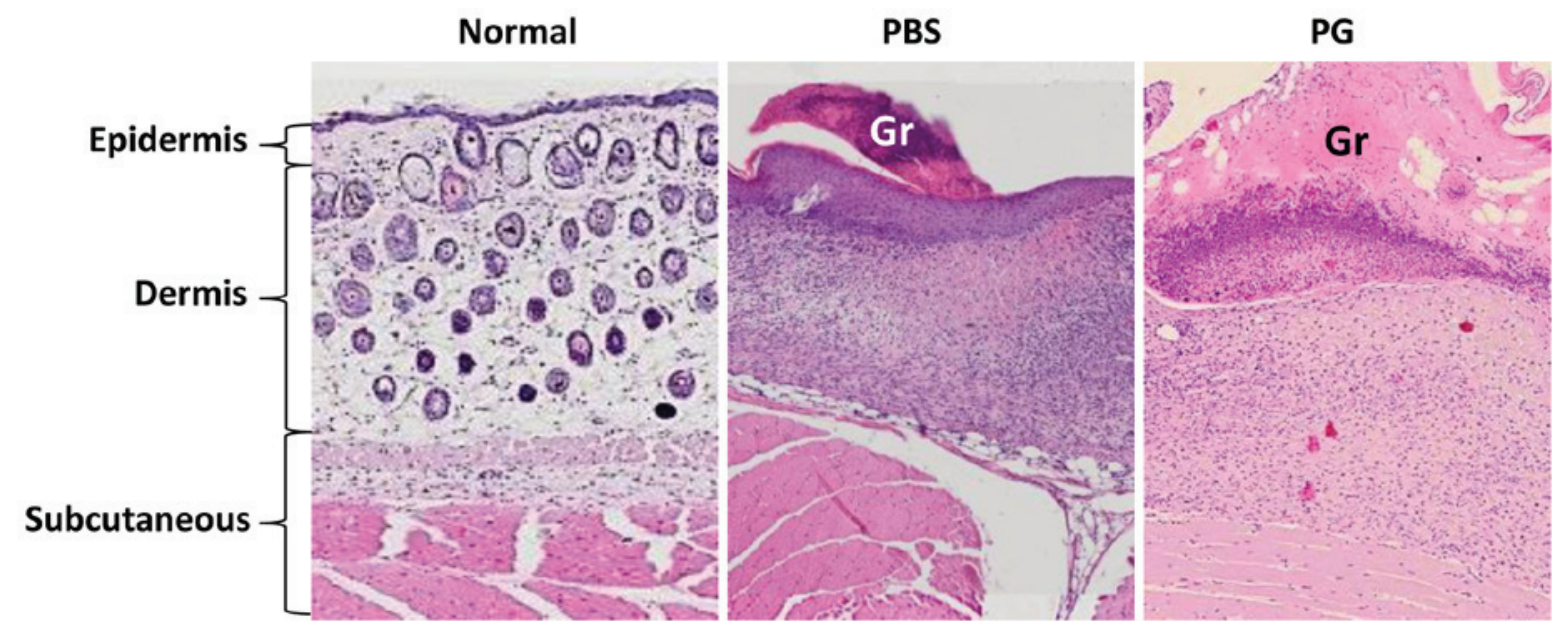

Figure 4. Histology of skin grafts. On day 9, sections of skin grafts were prepared and stained with hematoxylin and eosin (magnification, x40). The images are representatives of each group. PG, proteoglycan; PBS, phosphate-buffered saline; GR, graft. 
Our previous studies demonstrated that oral administration of PG inhibited recruitment of macrophages and neutrophils onto inflammatory sites via suppression of IL-17-induced chemokines in EAE (12) and collagen-induced arthritis (13). In the present study, reduction of cell infiltration under the graft was observed in PG-administered mice (Fig. 4). Therefore, prolonged survival of the grafts in $\mathrm{PG}$-administered mice may be due to inhibition of phagocyte recruitment through suppression of IL-17-induced chemokines, as shown in other mouse inflammatory models (11-14). To support this hypothesis, further evaluations of phagocytes, Th17 cells, IL-17, as well as other associated chemokines are required. In addition, molecular analysis of nuclear factor- $\kappa \mathrm{B}$ and Janus kinase/signal transducers and activators of transcription signaling on skin transplantation would be examined to provide an understanding of the molecular mechanism of PG.

To date, various immunosuppressant agents have been developed and used to prevent the rejection of transplanted organs or tissues (18). The majority of immunosuppressive agents act non-selectively, resulting in common side effects, including increased susceptibility to infections and decreased cancer immunosurveillance. Calcineurin inhibitors are associated with nephrotoxicity, cardiotoxicity and neurotoxicity (18), while everolimus induces stomatitis (19). These side effects affect the treatment course, such as discontinuation of therapy or dose reduction (19). To prolong the time before graft rejection during these irregular treatment courses, administration of mild immunosuppressive agents becomes an attractive option. Numerous natural compounds, such as non-digestible saccharides have been shown to suppress the immune response and promote health homeostasis (20). Fructooligosaccharides are found in various fruits and vegetables, and dietary supplementation with fructooligosaccharides attenuates allergic airway inflammation (21). However, to the best of our knowledge, their actions on graft rejection have not been examined.

In conclusion, in the present study, the suppressive effect of allograft rejection by PG was presented. Its action was not exhaustive, as the skin grafts were finally rejected in the PG-administered mice (Fig. 1), which indicates that PG is a mild anti-inflammatory substance. Therefore, salmon PG may present as a useful adjunct agent for immunosuppressant drugs. According to our previous studies, although PG demonstrates an anti-inflammatory effect, daily administration of PG did not affect bacterial infections or tumor growth in mice (unpublished data). Therefore, this biopolymer is considered to be a safe agent for prevention of graft rejection.

\section{Acknowledgements}

The present study was supported by the Ministry of Education, Culture, Sports, Science and Technology City Area Program for the Promotion of Science and Technology in Regional Areas (Japan).

\section{References}

1. Winsett R, Stratta RJ, Alloway R, Wicks MN and Hathaway DK: Immunosuppressant side effect profile does not differ between organ transplant types. Clin Transplant 15: 46-50, 2001.
2. Nankivell BJ, Borrows RJ, Fung CL-S, O'Connell PJ, Chapman JR and Allen RDM: Calcineurin inhibitor nephrotoxicity: longitudinal assessment by protocol histology. Transplantation 78: $557-565,2004$.

3. Schweer T, Gwinner W, Scheffner I, Schwarz A, Haller H and Blume C: High impact of rejection therapy on the incidence of post-transplant diabetes mellitus after kidney transplantation. Clin Transplant 28: 512-519, 2014.

4. Jiang S, Herrera O and Lechler RI: New spectrum of allorecognition pathways: implications for graft rejection and transplantation tolerance. Curr Opin Immunol 16: 550-557, 2004.

5. Yuan X, Paez-Cortez J, Schmitt-Knosalla I, D'Addio F, Mifarrej B, Donnarumma M, Habicht A, Clarkson MR, Iacomini J, Glimcher LH, et al: A novel role of CD4 Th17 cells in mediating cardiac allograft rejection and vasculopathy. J Exp Med 205: 3133-3144, 2008.

6. Gorbaxheva V, Fan R, Li X and Valujskikh A: Interleukin-17 promotes early allograft inflammation. Am J Pathol 177: 1265-1273, 2010

7. Itoh S, Nakae S, Axtell RC, Velotta JB, Kimura N, Kajiwara N, Iwakura Y, Saito H, Adachi H, Steinman L, et al: IL-17 contributes to the development of chronic rejection in a murine heart transplant model. J Clin Immunol 30: 235-240, 2010.

8. Wood KJ, Bushell A and Hester J: Regulatory immune cells in transplantation. Nat Rev Immunol 12: 417-430, 2012.

9. Danen EH and Yamada KM: Fibronectin, integrins, and growth control. J Cell Physiol 189: 1-13, 2001.

10. Sashinami H, Takagaki K and Nakane A: Salmon cartilage proteoglycan modulates cytokine responses to Escherichia coli in mouse macrophages. Biochem Biophys Res Commun 351: 1005-1010, 2006.

11. Mitsui T, Sashinami H, Sato F, Kijima H, Ishiguro Y, Fukuda S, Yoshihara S, Hakamada K and Nakane A: Salmon cartilage proteoglycan suppresses mouse experimental colitis through induction of Foxp3+ regulatory T cells. Biochem Biophys Res Commun 402: 209-215, 2010

12. Sashinami H, Asano K, Yoshimura S, Mori F, Wakabayashi K and Nakane A: Salmon proteoglycan suppresses progression of mouse experimental autoimmune encephalomyelitis via regulation of Th17 and Foxp3+ regulatory T cells. Life Sci 91: 1263-1269, 2012.

13. Yoshimura S, Asano $\mathrm{K}$ and Nakane A: Attenuation of collagen-induced arthritis in mice by salmon proteoglycan. Biomed Res Int 2014: 406453, 2014.

14. Hirose S, Asano K and Nakane A: Attenuation of obesity-induced inflammation in mice orally administered with salmon cartilage proteoglycan, a prophylactic agent. Biochem Biophys Res Commun 484: 480-485, 2017.

15. Billingham RE and Medawar PB: The technique of free skin grafting in mammals. J Exp Biol 28: 385-402, 1951.

16. Ye P, Rodriguez FH, Kanaly S, Stocking KL, Schurr J, Schwarzenberger P, Oliva P, Huang W, Zhang P, Zhang J, et al: Requirement of interleukin 17 receptor signaling for lung CXC chemokine and granulocyte colony-stimulating factor expression, neutrophil recruitment, and host defense. J Exp Med 194: 519-527, 2001.

17. Gaffen SL. An overview of IL-17 function and signaling. Cytokine 43: 402-407, 2008.

18. Mika A and Stepnowski P: Current methods of the analysis of immunosuppressive agents in clinical materials. J Pharm Biomed Anal 127: 207-231, 2016.

19. Ji YD, Aboalela A and Villa A: Everolimus-associated stomatitis in a patient who had renal transplant. BMJ Case Rep 2016: bcr2016217513, 2016.

20. Kerperien J, Jeurink PV, Wehkamp T, van der Veer A, van de Kant HJ, Hofman GA, van Esch EC, Garssen J, Willemsen LE and Knippels LM: Non-digestible oligosaccharides modulate intestinal immune activation and suppress cow's milk allergic symptoms. Pediatr Allergy Immunol 25: 747-754, 2014.

21. Yasuda A, Inoue KI, Sanbongi C, Yanagisawa R, Ichinose T, Yoshikawa $\mathrm{T}$ and Takano H: Dietary supplementation with fructooligosaccharides attenuates airway inflammation related to house dust mite allergen in mice. Int $\mathbf{J}$ Immunopathol Pharmacol 23: 727-735, 2010. 